\title{
Design and implementation of adversarial virtual simulation training system
}

\author{
Shixiong $\mathrm{Li}^{1,}{ }^{*}$, Xuwen Sun ${ }^{2}$, Hongchao Zhou ${ }^{2}$, Tianlei Zhang ${ }^{2}$, and Zhenjiang W ang ${ }^{2}$ \\ ${ }^{1}$ D ept. Surface V essels Command, China Coast Guard A cademy, Ningbo, China \\ ${ }^{2}$ D ept. Surface V essels Command, Dalian N aval A cademy, Dalian, China
}

Keywords: adversarial training, virtual simulation, system design.

\begin{abstract}
With a view to further enriching the college practical teaching conditions and also for the college teaching being closer to actual combat, a study is made on basis of system demand analysis in the early stage about the internal interface relationship and information/control process of the antagonistic virtual simulation training system with a key technology analysis made to lay a solid foundation for the implementation of the antagonistic virtual simulation training system.
\end{abstract}

\section{Introduction}

Antagonistic training is a group training method that can effectively improve and test comprehensive combat effectiveness ${ }^{[1]}$. Antagonistic training refers to the training which, based on the training purpose, has the participating forces divided into two sides against each other (as hypothetical enemy each other) with each side organizing and implementing the training in the form of offensive and defensive confrontation in a unified training system based on their own roles. ${ }^{[2]}$ With the further development of training practice and the requirement for training actual combativeness becoming increasingly higher, antagonistic training has exhibited multiple forms to adapt to the training requirements with different objects, different levels, different subjects. At the same time, the teaching activities based on antagonistic training are also an important means of college practice teaching. The virtual simulation training based on computer, due to such advantages as being more scientific in organization and implementation, more quantitative in judgment and evaluation, more realistic in battlefield simulation, closer to actual combat, etc., has become an important means of drill in today's advanced military countries. ${ }^{[3}$ It has become a very effective form and means of antagonistic training. The US troops also pays attention to antagonistic virtual simulation training. At present, there are at least 30 combat laboratories of different levels and types built ${ }^{[4]}$, including also an antagonistic virtual training system. On basis of the demand analysis and design of antagonistic virtual simulation training system in the early stage, the antagonistic virtual simulation training system has emerged at the right moment to enrich the guarantee conditions of antagonistic training teaching

* Corresponding author: coudutyonthesea@ 163.com 
activities in college. The training process of antagonistic virtual simulation includes three basic stages, i.e. training preparation, training implementation and training evaluation.

\section{Design of technical architecture}

The technical architecture system consists of device resource layer, data resource layer, cross-domain heterogeneous operation support layer, application layer and business layer from bottom to top, as shown in Fig. 1.

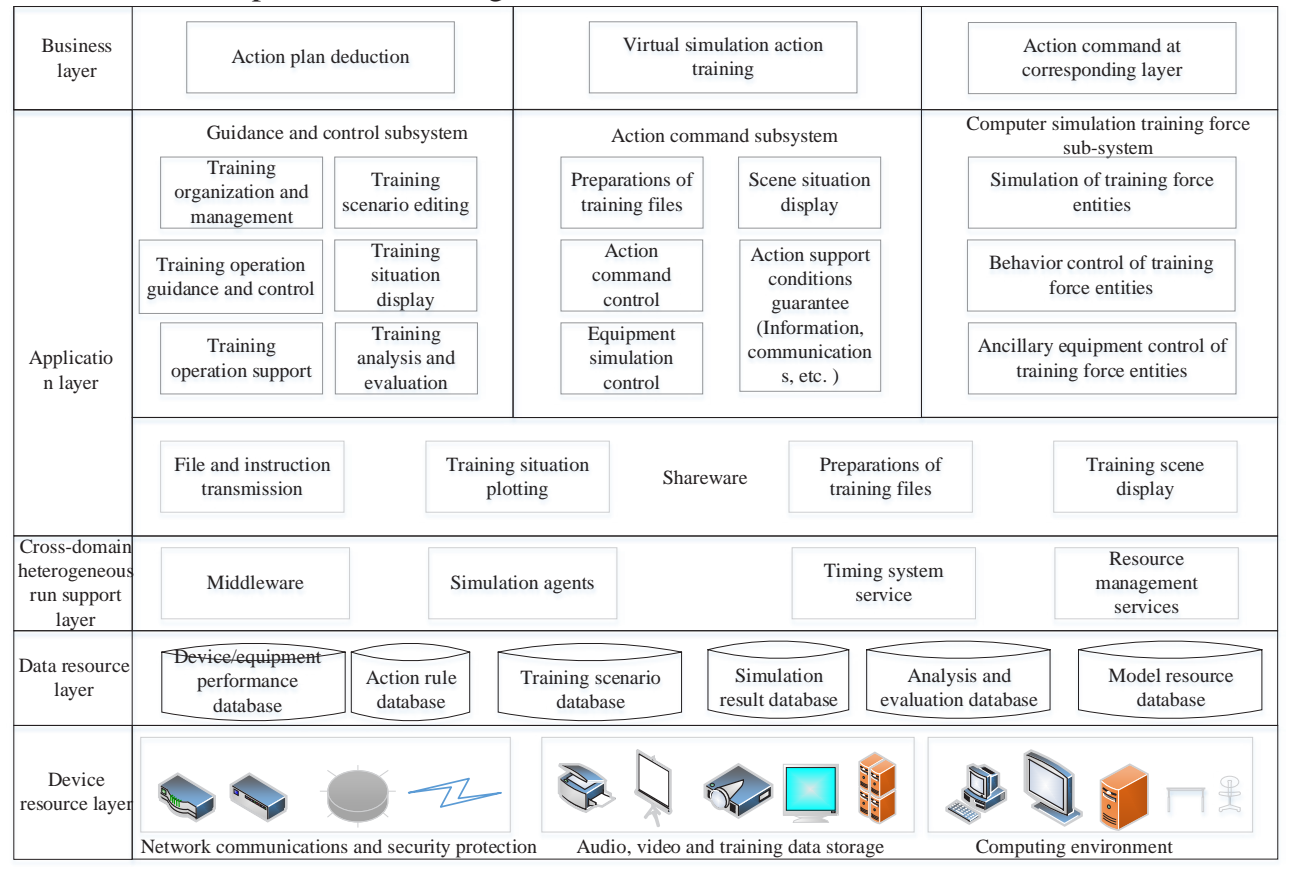

Fig. 1. Technical architecture of the system.

The device resource layer provides hardware equipment related to network communications and security protection, audio, video and storage-related hardware equipment, as well as computing environment-related hardware equipment. The data resource layer contains all kinds of databases related to training, such as device/equipment performance database, action rule database, training scenario database, model resource database, etc., which are used to store training data, file resources and so on and provide data support and storage services for the operation of training system and training analysis and evaluation. The cross-domain heterogeneous operation support layer, aiming to meet the interconnection requirements of heterogeneous simulation systems and installed simulators, based on distributed simulation architecture, solves the interconnection and interaction problems of application systems based on real installation, simulators and simulation systems by using network, distributed computing and middleware, etc. Relying on the communication infrastructure, it builds interconnection and integration services of systems, provides middleware, timing system services, simulation agents, resource management and other service functions, and supports the integration, operation and management of training application systems that integrate virtuality and reality and are consistent with time and space. The application layer consists of the guidance and control subsystem, the action command system (corresponding command levels are set according to the requirements of the training actions) and the computer simulation training force 
subsystem. It provides guidance and modulation control for virtual simulation training, grouping and management of training forces, as well as action command for training forces. The business layer specifically provides support for requirements of such businesses as action plan deduction, virtual simulation action training and action command at corresponding levels. ${ }^{[5]}$

\section{Internal interface relationship of the system}

The internal interface relationships of the system mainly include the information transmission and control relationships between such functional modules as training organization and management, training scenario edition, training operation management and control, training process introduction, training operation support, training situation display, training data collection, training process playback, training analysis and evaluation, training effect decision and so on, command stations at all levels, computer simulation model of training forces and databases. Taking the adversarial training with three-level command relationships as an example, the interface relationship between functional modules within the system is shown in Fig. 2.

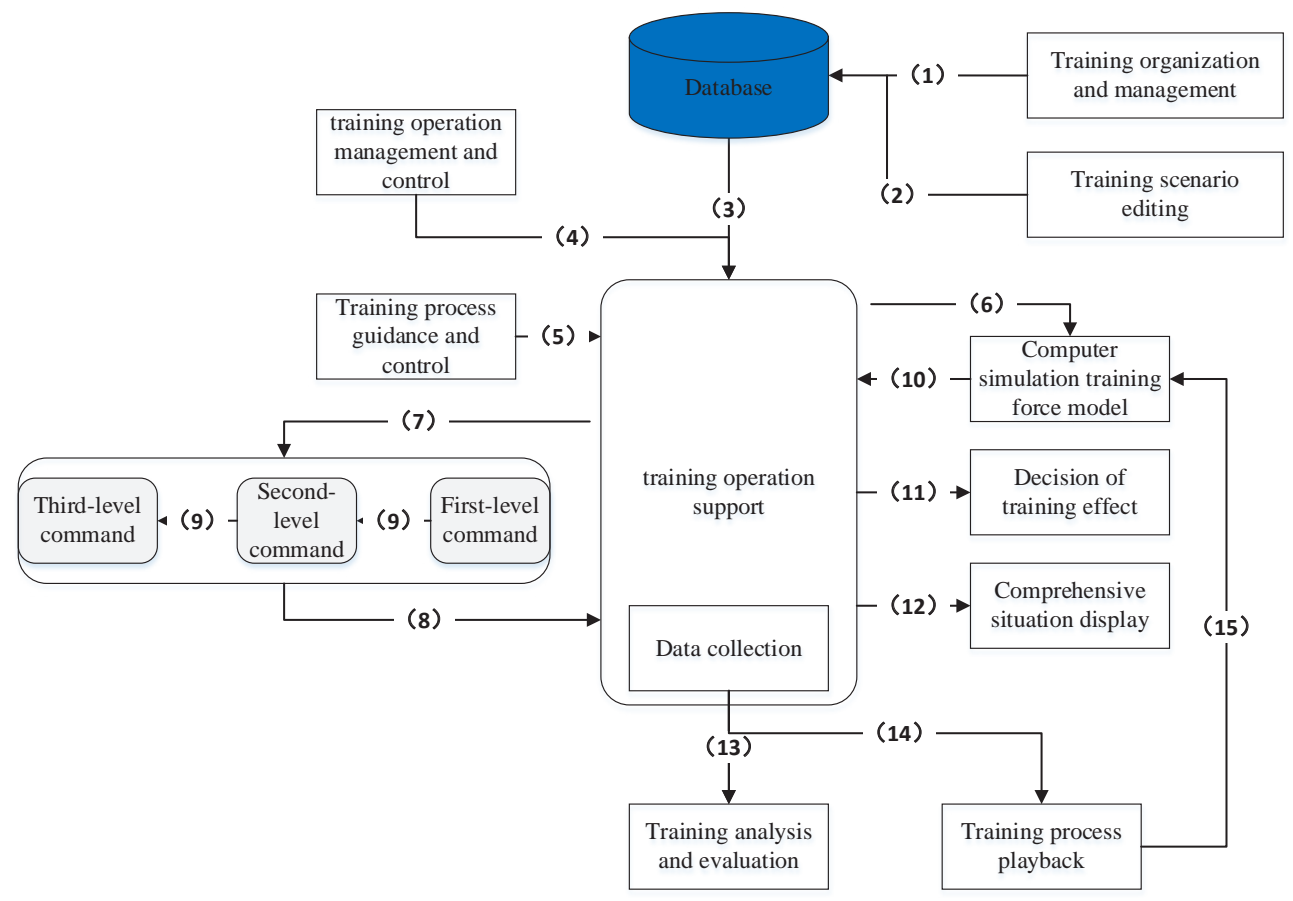

Fig. 2. Internal interface relationships of the system.

The types of information involved in each interface in the figure are as follows:

(1) Training organization and management information: the training organization and management module completes it and provides training task information, training role information and training data, etc.

(2) Training scenario data: the training scenario edition module completes the setup of training plots, grouping and deployment of training entities, establishes relationships between entities, and stores them in the resource database for use when scenario is loaded. 
(3) Training configuration information: the simulation resource database provides information including model data, scenario data, system operation configuration and so on for use when the simulation engine is loaded.

(4) Operation control instructions: the instructions that control the start, pause, continuation and stop of training activities;

(5) Training process guidance and control instructions: instructions to conduct raining process adjustment, training force conditioning, device/equipment conditioning and other related instructions;

(6) Computer simulation training force guidance and control instructions: including generation control instructions, conditioning instructions, simulation command instructions and situation information of CGF training entities;

(7) Conditioning instructions and situation information of the command stations: situation information and conditioning command information provided by the simulation engine to the command stations (at all levels);

(8) Status information of command stations: the status information of simulation engine returned by the command stations (at all levels);

(9) Command information: the command instruction information sent from the higher command station to the lower command one;

(10) Computer simulation training force situation information: the status information of CGF training entities;

(11) Decision: Decision information of adversarial training;

(12) Comprehensive situation: the global situation information provided by the simulation engine, which is used as the situation information of the guidance and control station;

(13) Training evaluation data: data collected by the data acquisition module for training evaluation.

(14) System playback files: playback files in the entire training process;

(15) C GF playback data: action data file of CGF training entities for training playback.

\section{Information/control process of the system}

According to the three basic stages (training preparation, training implementation and training evaluation) included in the virtual simulation training process, the information/control process of the system is constructed based on the interface relationships between functional modules of the system, as shown in Fig. 3. 


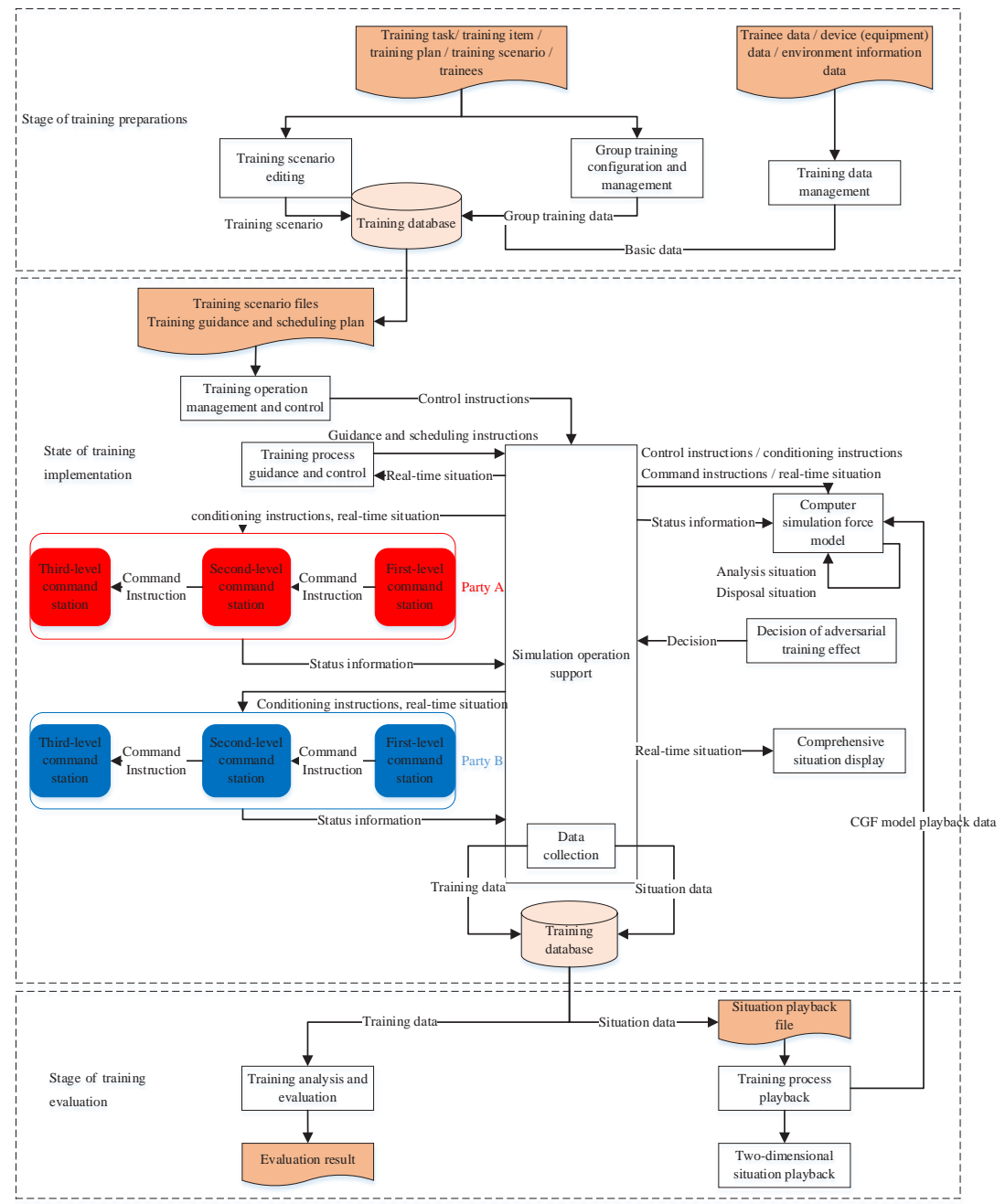

Fig. 3. Information / control process of the system.

\section{Conclusion}

To construct the adversarial virtual simulation training system based on computer virtual simulation technology, it is necessary to clarify the general organizational process of adversarial virtual training, scientifically construct the technical architecture of the system, define the functional composition of the system, rationally construct the internal interface relationships of the system, as well as the information/control process of the system. In addition, the scalability of the model and the scalability of the system application functions should be fully considered to ensure the adaptability of the system to different users and different training tasks, and better meet the requirements for the transformation and upgrading of various models caused by the changes in device/equipment, training force, action style and training environment. Therefore, it is necessary to pay attention to a series of key technical issues closely related to the system construction to ensure that the system construction has a good technical support. It is hoped that this study can provide useful reference for the construction of adversarial virtual simulation training system. 


\section{References}

1. Wang Xiaohui. A Brief Study on A ctual Combat Teaching in the College Where Office Is Take [J]. Journal of Shijiazhuang M echanized Infantry Institute, 2014(2):19 $-20$

2. Committee for the A pproval of Psychological Terms. Chinese Terms in Psychology (2nd Edition) [M ]. B eijing: Science Press, 2019.02.

3. X $\mathrm{i}$ Zan, Dong Kai. A nalysis on the Development of Virtual Simulation Training in M ilitary Field under the V iew of Globalization, 2017(3):63-64

4. $\quad H$ Gao Biao. A Brief Analysis to the Characteristics of A ntagonistic Training in US Troops [J]. J ournal of A rmed Police Command Institute, 2017, 23(10):62-63

5. $Y$ unzhi $Y u$, Jianqing Cheng, $Y$ ufang Zhou. Research on Simulation Training Technology of Combat Command A utomation System [J]. Ship Electronic Engineering, 2008,(07): 118-122 